
\title{
25 Research Soure \\ Uveitis Including Vogt-Koyanagi-Harada Syndrome Following Inactive Covid-19 Vaccination: A Case Series
}

\section{Mehrdad Motamed Shariati}

Mashhad University of Medical Sciences

Mojtaba Abrishami

Mashhad University of Medical Sciences

Shahin Jahani

Mashhad University of Medical Sciences

Ali Bolouki

Mashhad University of Medical Sciences

Mohamadreza Ansari Astaneh

Mashhad University of Medical Sciences

Seyedeh Maryam Hosseini ( $\sim$ Smaryam_hosseini@yahoo.com )

Mashhad University of Medical Sciences

\section{Research Article}

Keywords: Coronavirus disease 2019, COVID-19 vaccination, Severe acute respiratory syndrome coronavirus 2, Sinopharm vaccine, Uveitis, Vogt-Koyanagi-Harada

Posted Date: March 1st, 2022

DOI: https://doi.org/10.21203/rs.3.rs-1393405/v1

License: (9) This work is licensed under a Creative Commons Attribution 4.0 International License. Read Full License 


\section{Abstract}

Background: Currently, large populations around the world have been vaccinated against COVID-19. The whole inactivated Sinopharm COVID-19 vaccine has been the main available and widely used COVID-19 vaccine in Iran. Ocular inflammatory reactions have been reported following vaccination. This case report aimed to introduce four cases of uveitis after Sinopharm vaccine inoculation.

Case presentation: The first case was a 38-year-old woman who was a known case of ulcerative colitis whose systemic disease was inactive during the past four years. Active uveitis was developed following the second dose of the COVID-19 vaccination. The other three cases were completely healthy individuals who developed the first episode of uveitis, including one case of Vogt-Koyanagi-Harada syndrome, after the COVID-19 vaccination. All cases responded well to corticosteroid treatments.

Conclusion: our cases and ongoing reports around the world raise concerns about the possibility of uveal inflammations in cases with a history of auto-immune systemic diseases or inactive uveitis after the COVID-19 vaccination.

\section{Introduction}

As an ongoing global pandemic, COVID-19 is a contagious respiratory disease caused by severe acute respiratory syndrome coronavirus 2 (SARS-COV-2). Vaccination is one of the most effective ways to control the infection. The whole inactivated COVID-19 Sinopharm vaccine has been the main available and widely used COVID-19 vaccine in Iran.

There have been reports of the incidence of inflammatory eye diseases following Sinopharm vaccination. El Sheikh et al. reported an 18-year-old female case of juvenile idiopathic arthritis with bilateral anterior uveitis following the second dose of the Sinopharm vaccine. ${ }^{1}$ Lijie Pan et al. reported a case of bilateral posterior uveitis five days after inoculation with inactivated COVID-19 vaccine, who showed a good response to local and systemic steroids. ${ }^{2}$ Goyal et al. introduced a 34-year-old male with large macular serous detachment and choroidal thickening one week after the second dose of the COVID-19 vaccine. The patient was treated with oral corticosteroid and improved rapidly. ${ }^{3}$ It is not known whether the vaccine stimulates the immune system and causes ocular inflammation or this process is merely coincidental. We hereby present four cases with uveitis following the inactivated COVID-19 vaccination.

\section{Case Presentation}

\section{Case1}

A 38-year-old woman, known case of ulcerative colitis for 20 years, whose systemic disease was inactive during the past four years (despite taking no medication and having no obvious history of ocular inflammation) was referred to the emergency department of Khatam-Al-Anbia Eye Hospital, affiliated with Mashhad University of Medical Sciences, Mashhad, Iran. The patient had complaints of blurred vision, 
ocular pain, and photophobia in her left eye six days after inoculation with the second dose of Sinopharm vaccine. She had no other ocular or systemic symptoms. The best-corrected visual acuity (BCVA) was 20/32 for her left eye. The anterior segment examination of the left eye showed posterior synechia, ciliary injection, fine keratic precipitates (KPs), 4+ anterior chamber (AC) cells and flare, and 1+ vitreous cell, according to SUN Working Group classification ${ }^{4}$. Intraocular pressure was within the normal limit in both eyes. Fundus examination, macular optical coherence tomography (OCT), and fundus autofluorescence (FAF) revealed no further abnormality (Figure 1). Rheumatologic and gastroenterology consults were requested, and treatment was started with oral prednisolone (25 mg/day), topical corticosteroid, and cycloplegic medication. The rheumatologic consult was unremarkable; however, colonoscopy showed active ulcerative lesions. Azathioprine, mesalamine and folic acid were initiated. After 10 days of treatment, BCVA increased to $20 / 20$, AC reaction reduced to +0.5 , and posterior synechia was broken. Oral and topical steroids were tapered off over four weeks and no relapse was observed during a 3-month follow-up.

\section{Case 2}

The second case was a 15-year-old female who developed red-eye, photophobia, and blurred vision bilaterally three days after inoculation with the first dose of the Sinopharm vaccine. We examined the patient two days later. The patient's BCVA was 20/25 for both eyes, and slit-lamp examination revealed bilateral ciliary injection, symmetrical 4+AC cell, and 2+ flare in both eyes. The past medical, ocular, and drug histories were unremarkable, and systemic workup results (e.g., hematologic and serologic tests) were inconclusive. Macular OCT and fluorescein angiography (FA) showed no other pathological findings (Figure 2). We prescribed frequent topical corticosteroids and cycloplegics. After four days of treatment, the ciliary injection and $A C$ reaction of the patient were significantly reduced and her BCVA increased to $20 / 20$ in both eyes. Treatment was slowly tapered off for two weeks, and inflammation was alleviated in the last 3-month follow-up.

\section{Case 3}

A 65-year-old male was referred to our clinic with complaints of blurred vision and photophobia 12 days after inoculation with the second dose of Sinopharm vaccine. The patient reported malaise and anorexia after injection of the second vaccine dose. Eye redness, pain, photophobia, and blurred vision occurred six days later. Slit-lamp examination showed $3+A C$ cells and $1+$ vitreous cells in the right eye and 2+AC cells and no vitreous cell in the left eye. Fundus examination revealed disc hyperemia and two small patches of retinitis less than two disc diameters in the posterior pole of the right eye (Figure 3 ). Fundus examination of the left eye was normal. Vitreous sampling was performed with a 25-gauge needle through pars-plana and evaluated using polymerase chain reaction (PCR) to detect the Herpes Simplex virus, Varicella Zoster virus, Cytomegalovirus, and Toxoplasma. A thorough systemic work-ups and rheumatology consultation was requested subsequently. Following negative results of ocular PCR for microbes, nasopharyngeal PCR for COVID-19, and systemic work-ups oral prednisolone ( $25 \mathrm{mg} / \mathrm{day})$ was started in addition to topical corticosteroid and cycloplegics. In the follow-up visit two weeks later, AC 
reaction reduced to $1+c e l l$ in both eyes, and the retinitis patches were resolved. Oral and topical steroids slowly tapered off within four weeks.

\section{Case 4}

A 23-year-old female patient with no previous medical or medication history was referred to our clinic for evaluation of progressive vision loss. She had received a second dose of Sinopharm vaccine two weeks ago and reported episodes of malaise and fever for one week. One week after inoculation, the patient had experienced severe headaches and the vision of both eyes started to decline. Topical steroid drops had been administered to her for bilateral anterior uveitis with no effect, and she was then referred to our clinic for further evaluation. BCVA was $20 / 100$ for the right eye and 20/630 for the left eye. Slit-lamp examinations revealed fine KPs, 3+ AC cells, 1+ flare, and 1-2+ vitreous cells in both eyes. Fundus examination showed bilateral disc hyperemia and multifocal posterior pole serous retinal detachment in both eyes. FAF, FA, and indocyanine angiography images are presented in Figure 4. Macular OCT revealed severe serous retinal detachment in both eyes with multiple septae, heterogenous hyperreflective subretinal material, and undulations of the thickened choroid in favor of Vogt-Koyanagi-Harada (VKH) syndrome. Moreover, severe disruption of the outer retinal layers was noted (Figure 5). The patient was admitted with the diagnosis of probable VKH syndrome, and intravenous methylprednisolone (1 $\mathrm{gr}$ per day), topical steroid, and cycloplegic were administrated accordingly. After three days of treatment, BCVA increased to 20/50 OD and 20/500 OS, and a significant reduction of subretinal fluid was observed in the OCT images. The intravenous steroid was discontinued and the patient was discharged with oral prednisolone $(1 \mathrm{mg} / \mathrm{kg} /$ day $)$.

At the last F/U three weeks after treatment, BCVA was 20/20 and 20/30 in the right and left eye, respectively.

\section{Discussion}

Vaccine-related uveitis is a well-established and well-documented phenomenon and has been reported in relation to all available vaccines. The exact pathogenesis has remained unclear but molecular mimicry, antigen-specific cell, and antibody-mediated hypersensitivity reactions are the most recognized hypothesis. ${ }^{5}$

Reports of post-COVID-19 immune-mediated diseases, such as uveitis, Guillain-Barre syndrome, or systemic lupus erythematosus have been published. It is postulated that COVID-19, the same as other viruses, can disturb self-tolerance and incite autoimmune responses through cross-reactivity with host cells. The same immunologic process may occur following vaccination ${ }^{6}$.

In the current case report, we presented four cases of uveitis, including a case of VKH syndrome, after inoculation with inactivated COVID-19 Sinopharm vaccine. The first case was a patient with unilateral anterior uveitis and reactivated ulcerative colitis six days after inoculation with the second dose of Sinopharm vaccine. The patient's underlying disease was inactive for four years without any medication. 
Ocular manifestations responded well to topical and systemic steroids, and systemic medications were initiated for treating active ulcerative colitis. Case 2 presented the onset of bilateral anterior uveitis in an otherwise healthy female with no ocular history. Inflammation was completely resolved after topical treatment and systemic workup was unremarkable. The third case developed panuveitis with two foci of posterior polar retinitis patches in the right eye and isolated anterior uveitis in the left eye. The patient responded to the combination of oral and topical steroids with complete resolution of uveitis and retinitis patches.

The last case in this series was a young female diagnosed with probable VKH syndrome after inoculation with the second dose of inactivated vaccine. VKH is a multisystem immune-mediated disease presenting with bilateral, non-necrotizing granulomatous panuveitis, and serous retinal detachment associated with neurological, auditory, and/or dermatological disorders. ${ }^{7} \mathrm{VKH}$ is a T-cell mediated immune response triggered by environmental or immunogenetic agents; however, its exact etiology that primarily involves the choroid is not recognized precisely. Genetic susceptibility and viral disease are probable risk factors of $\mathrm{VKH}^{8}$

There have been multiple reports of VKH syndrome following vaccination for influenza ${ }^{9}$, yellow fever ${ }^{10}$, and Bacillus Calmette-Guérin ${ }^{11}$. Papasavvas et al. reported reactivation of previously diagnosed and well-controlled VKH for the past six years and six weeks after inoculation with the second dose of the BNT162b2 vaccine. ${ }^{12}$ Saraceno et al. described the onset of bilateral VKH in a 62-year-old healthy female following vaccination with Oxford-AstraZeneca chimpanzee adenovirus vectored vaccine ${ }^{13}$. To the best of our knowledge, this is the first reported case of VKH following vaccination with the inactivated vaccine of Sinopharm.

It is postulated that dysregulation of the immune system and molecular mimicry is the possible etiologies of uveitis following vaccination. Xin Le Ng et al. reviewed the ocular side effects of the COVID-19 vaccination. Regarding the overlap between the ocular manifestations of the vaccination and the COVID19 itself, researchers suggested a possible similar mechanism ${ }^{14}$.

The appropriate response to corticosteroid treatment is the common denominator of all the introduced cases. The prompt response confirms that despite the possibility of stimulating the immune system after vaccination, inflammation responds well to treatment without any significant vision-threatening complications, regardless of the type of uveitis. Therefore, awareness of ophthalmologists about complications of the COVID-19 vaccine is important for reassuring patients regarding the benign course of inflammation and conducting specific research to identify the possible stimulating particle of the COVID-19 vaccine in the near future. One plausible mechanism of immunogenicity of the vaccine may be related to the adjuvant particles of vaccines that trigger immunologic reaction via multiple mechanisms, including antigen-presenting-cells activation, cytokine release, and sustained release of antigen due to depo effect of adjuvants, antibody production, and cellular recruitment. ${ }^{15}$ 
Another important mechanism can be related to the existence of specific viral particles in vaccines, including the COVID-19 vaccine, which causes an immunologic reaction in the body, including uveal tissues as a potent immunogenic tissue in the eye.

It should be noted that no definite causal relationship can be established based on the cases in this report, and the possible ocular side effects are rare despite the large scale of worldwide vaccination. However, our cases and ongoing reports around the world raise concerns about the possibility of uveal inflammations in cases with a history of auto-immune systemic diseases or inactive uveitis after the COVID-19 vaccination.

\section{Declerations}

\section{Ethics approval and consent to participate}

This study followed the tenets of the Declaration of Helsinki, and the Mashhad University of Medical Sciences ethical committee approved this study.

\section{Consent for publication}

We obtained informed consent from the patient to publish his examination results and images.

\section{Conflict of interest}

The authors declare any conflict of interests with the results of this paper.

\section{Authors' contributions}

Conceptualization: Mehrdad Motamed Shariati, Seyedeh Maryam Hosseini.

Patient examination and imaging: Seyedeh Maryam Hosseini, Mohammadreza Ansari Astaneh, Mojtaba Abrishami, Shahin Jahani, Mehrdad Motamed Shariati

Writing the manuscript: Mehrdad Motamed Shariati, Seyedeh Maryam Hosseini, Ali Bolouki

Supervision: Seyedeh Maryam Hosseini, Mehrdad Motamed Shariati

\section{Availability of data and materials}

The datasets used during the current study are available from the corresponding author on reasonable request

\section{Funding}

The authors received no funding. 


\section{References}

1. ElSheikh RH, Haseeb A, Eleiwa TK, Elhusseiny AM. Acute uveitis following COVID-19 vaccination. Ocular Immunology and Inflammation. 2021 Aug 8:1-3.

2. Pan $L$, Zhang Y, Cui Y, Wu X. Bilateral uveitis after inoculation with COVID-19 vaccine: A case report. International Journal of Infectious Diseases. 2021 Sep 30.

3. Goyal M, Murthy SI, Annum S. Bilateral multifocal choroiditis following COVID-19 vaccination. Ocular immunology and inflammation. $2021 \mathrm{Jul}$ 28:1-5.

4. Standardization of Uveitis Nomenclature (SUN) Working Group. Standardization of uveitis nomenclature for reporting clinical data. Results of the First International Workshop. American journal of ophthalmology. 2005 Sep 1;140(3):509-16.

5. Emmett T. Cunningham Jr, Ramana S. Moorthy, Frederick W. Fraunfelder \& Manfred Zierhut (2019) Vaccine-Associated Uveitis, Ocular Immunology and Inflammation, 27:4, 517-520.

6. Liu Y, Sawalha AH, Lu Q. COVID-19 and autoimmune diseases. Current Opinion in Rheumatology. 2021 Mar;33(2):155.

7. O'Keefe GA, Rao NA. Vogt-koyanagi-harada disease. survey of ophthalmology. 2017 Jan 1;62(1):125.

8. Chi W, Yang P, Li B, Wu C, Jin H, Zhu X, Chen L, Zhou H, Huang X, Kijlstra A. IL-23 promotes CD4+ T cells to produce IL-17 in Vogt-Koyanagi-Harada disease. Journal of allergy and clinical immunology. 2007 May 1;119(5):1218-24.

9. Yoshino N, Kawamura A, Ishii A, Yoshida K, Watanabe T, Yamashita T, Fukuchi T, Toyoda F, Kakehashi A, Sugawara H. Vogt-Koyanagi-Harada Disease Associated with Influenza A Virus Infection: A Case Report. Internal Medicine. 2018:9819-17.

10. Campos WR, Cenachi SP, Soares MS, Gonçalves PF, Vasconcelos-Santos DV. Vogt-KoyanagiHarada-like disease following yellow fever vaccination. Ocular immunology and inflammation. 2021 Jan 2;29(1):124-7.

11. Dogan B, Erol MK, Cengiz A. Vogt-Koyanagi-Harada disease following BCG vaccination and tuberculosis. Springerplus. 2016 Dec;5(1):1-1.

12. Papasavvas I, Herbort CP Jr. Reactivation of Vogt-Koyanagi-Harada disease under control for more than 6 years, following anti-SARS-CoV-2 vaccination. J Ophthalmic Inflamm Infect. Jul 5, 2021;11(1):21.

13. Saraceno JJF, Souza GM, Dos Santos Finamor LP, Nascimento HM, Belfort R Jr. Vogt-KoyanagiHarada Syndrome following COVID-19 and ChAdOx1 nCoV-19 (AZD1222) vaccine. Int J Retina Vitreous. 2021 Aug 30;7(1):49.

14. Ng XL, Betzler BK, Testi I, Ho SL, Tien M, Ngo WK, Zierhut M, Chee SP, Gupta V, Pavesio CE, de Smet MD. Ocular adverse events after COVID-19 vaccination. Ocular immunology and inflammation. 2021 Sep 26:1-9. 
15. Kim M. Vogt-Koyanagi-Harada syndrome following influenza vaccination. Indian journal of ophthalmology. 2016;64(1).

\section{Figures}

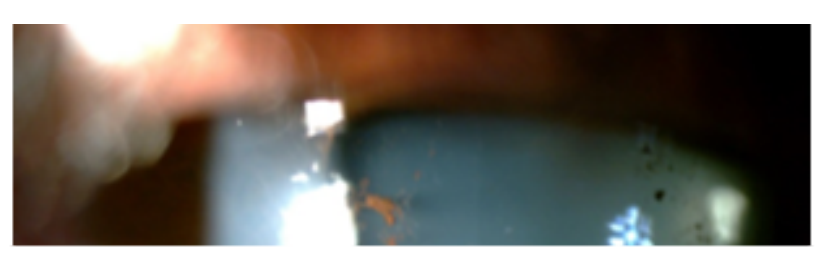

\section{Figure 1}

Slit photo of the left eye shows diffuse KP's, posterior synechia, pigment on the crystalline lens and anterior chamber reaction. Macular OCT and FAF of the involved eye show no abnormality. 


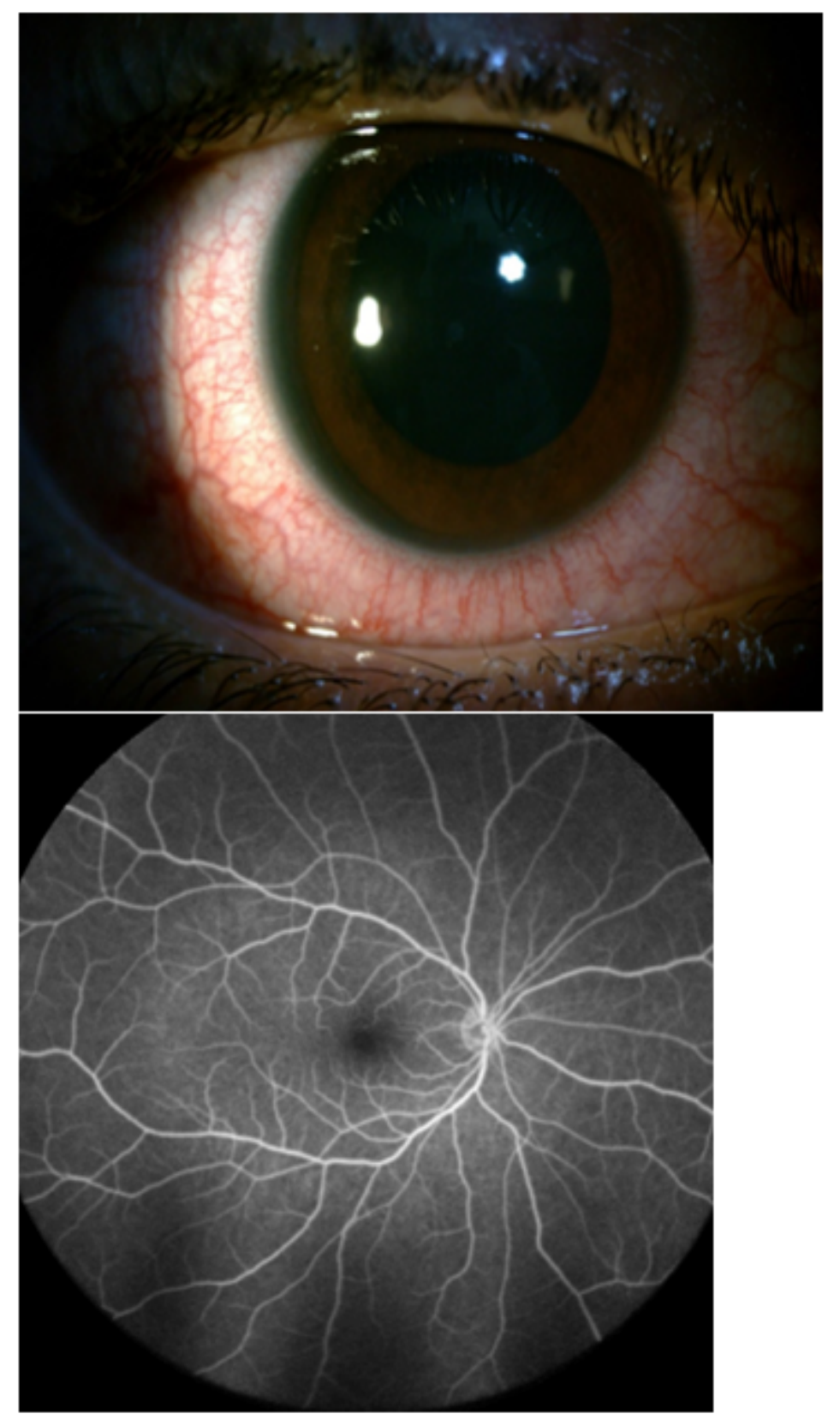

Figure 2

Slit photo of Right eye shows ciliary injection. Fluorescein angiography shows no further pathology. 


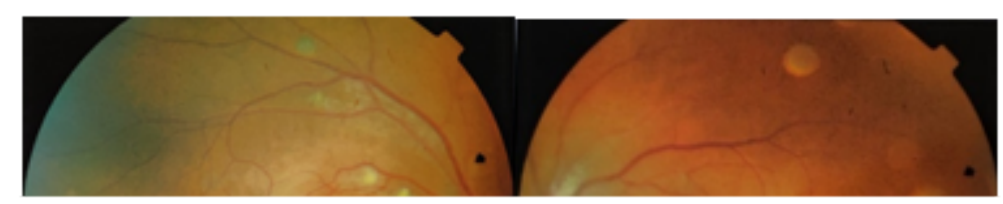

\section{Figure 3}

Fundus photo of the right eye shows disc hyperemia and two small patches of retinitis; Fundus photo of the left eye is normal. Fundus autofluorescence (FAF) shows hypo autofluorescence in the same location of retinitis patches. Fluorescein angiography showed no vascular leakage. Optical Coherence Tomography (OCT) of the right eye reveals increased inner retinal thickness and reflectivity at the site of the retinitis patches.

\section{Figure 4}

FAF shows hyper autofluorescence in the macula with hypo autofluorescence in the areas of the serous detachment; FAG reveals multiple areas of pinpoint leakage in the posterior pole. ICGA demonstrates multiple dark spots in the mid-phase angiogram. 


\section{Figure 5}

Macular OCT before treatment revealed severe serous retinal detachment in OU with multiple septae, heterogenous hyperreflective subretinal materials, and undulations of the thickened choroid. Also, severe disruption of outer retinal layers was noted. OCT in day 3 shows significant reduction of subretinal fluid and choroidal undulations.

\section{Figure 6}

macular oct after treatment shows decrease subretinal fluid . 